\title{
Влияние быстрого термического отжига на распределение атомов азота в GaAsN/GaAs
}

\author{
(C) А.А. Лазаренко ${ }^{1}$, К.Ю. Шубина ${ }^{1}$, Е.В. Никитина ${ }^{1,2}$, Е.В. Пирогов ${ }^{1}$, \\ А.М. Мизеров ${ }^{1}$, М.С. Соболев ${ }^{1}$ \\ ${ }^{1}$ Санкт-Петербургский национально-исследовательский \\ Академический университет им. Ж.И. Алфёрова Российской академии наук, \\ 194021 Санкт-Петербург, Россия \\ 2 Физико-технический институт им. А.Ф. Иофффе Российской академии наук, \\ 194021 Санкт-Петербург,Россия \\ E-mail: alexashpigun@yandex.ru
}

Поступила в Редакцию 8 июня 2021 г.

В окончательной редакции 21 июня 2021 г.

Принята к публикации 21 июня 2021 г.

Исследуется влияние быстрого термического отжига тройных твердых растворов $\mathrm{GaAs}_{1-x} \mathrm{~N}_{x} / \mathrm{GaAs}$ на распределение атомов азота в кристаллической решетке. Образцы исследуются методами фотолюминесценции и высокоразрешающей рентгеновской дифрактометрии. Из-за несоответствия размеров и электроотрицательности атомов азота и мышьяка, азот встраивается неравномерно в кристаллическую решетку GaAs. Показаны варианты расположения атомов азота в кристаллической решетке GaAs до и после быстрого термического отжига.

Ключевые слова: разбавленные нитриды, гетероструктуры, молекулярно-пучковая эпитксия, арсенид галлия, азот.

DOI: 10.21883/FTP.2021.11.51563.9697

\section{1. Введение}

GaAs является одним из самых важных и распространенных материалов микроэлектроники. В настоящее время существует большой интерес к увеличению области применения материала GaAs. В частности, интерес представляет добавление азота в $\mathrm{GaAs}$ для получения азотосодержащего твердого раствора $\mathrm{GaAs}_{1-x} \mathrm{~N}_{x}$, который является перспективным материалом для применения в солнечных элементах [1], лазерных устройствах [2] и спинтронике [3].

Классические тройные и четверные твердые растворы $\mathrm{A}^{\mathrm{III}} \mathrm{B}^{\mathrm{V}}$ могут быть выращены в широком диапазоне составов, и их электронные свойства плавно меняются с составом. В отличие от них разбавленные нитриды, например $\mathrm{GaAs}_{1-x} \mathrm{~N}_{x}$, возможно выращивать только в узком диапазоне составов, при этом добавление даже небольшого количество азота в матричный кристалл приводит к значительному изменению электронных свойств полученного твердого раствора [4]. Поэтому интерес представляет добавление азота в $\mathrm{GaAs}$, который позволяет значительно варьировать ширину запрещенной зоны $\mathrm{GaAs}_{1-x} \mathrm{~N}_{x}$ при небольшом изменении состава $[4,5]$.

При эпитаксиальном росте твердого раствора $\mathrm{GaAs}_{1-x} \mathrm{~N}_{x}$ атомы азота заменяют атомы мышьяка в кристаллической решетке. Однако значительная разница в размерах и электроотрицательности атомов азота и мышьяка приводит к неравномерному встраиванию атомов азота в кристаллическую решетку. Это приводит к возникновению локализованных состояний азота, а также нарушению трансляционной симметрии кристалла [4]. В связи с этим наиболее перспективная концентрация азота в соединении $\mathrm{GaAs}_{1-x} \mathrm{~N}_{x}-<2 \%$. При больших концентрациях происходит резкое ухудшение оптоэлектронных свойств таких твердых растворов [6].

Тем не менее даже при концентрациях азота $<2 \%$ в $\mathrm{GaAs}_{1-x} \mathrm{~N}_{x}$ наблюдается достаточно большое количество кристаллических дефектов, отрицательно влияющих на оптические и электронные свойства слоев [7].

Известно, что для улучшения кристаллического и оптического качества эпитаксиальных слоев часто используют быстрый термический отжиг [8].

В статье исследуется влияние быстрого термического отжига слоев GaAsN/GaAs на распределение атомов азота в кристаллической решетке.

\section{2. Эксперимент}

Объемные слои $\mathrm{GaAs}_{1-x} \mathrm{~N}_{x}$ толщиной 200 нм были выращены на полуизолирующих подложках $\mathrm{GaAs}$ (100) методом молекулярно-пучковой эпитаксии. Для получения атомарного азота использовался высокочастотный плазменный источник азота. Концентрация азота определялась по данным высокоразрешающей рентгеновской дифрактометрии на установке ДРОН 8 методом $\theta-2 \theta$-сканирования (рис. 1$)$.

На рис. 1 слева наблюдается рефлекс от подложки GaAs (100), справа от него - рефлексы от слоя $\mathrm{GaAs}_{0.9965} \mathrm{~N}_{0.0135}$ (красный) и $\mathrm{GaAs}_{0.9935} \mathrm{~N}_{0.0165}$ (синий). 


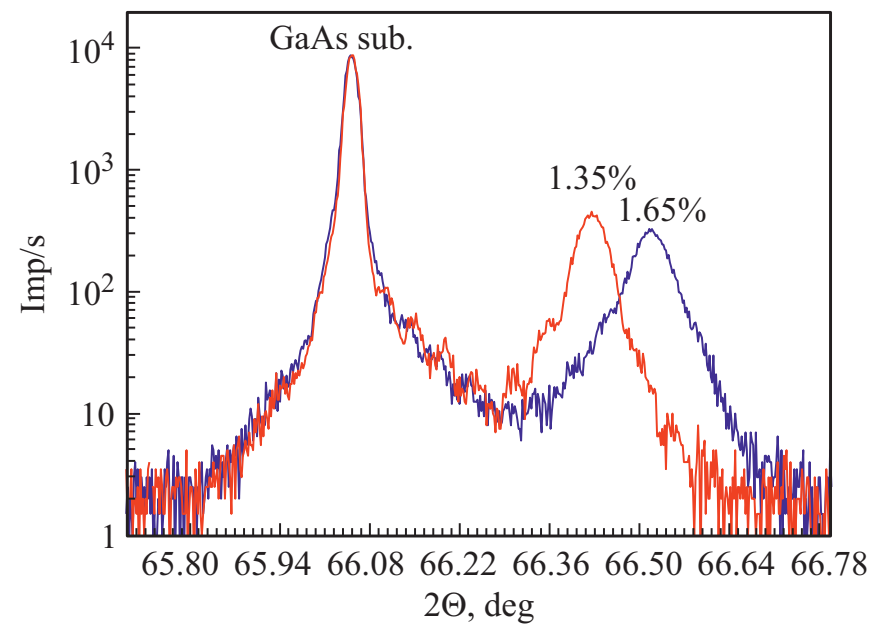

Рис. 1. Рентгенодифракционные кривые качания около симметричного рефлекса $\mathrm{GaAs}$ (004) от слоев $\mathrm{GaAs}_{0.9965} \mathrm{~N}_{0.0135}$ (красный) и $\mathrm{GaAs}_{0.9935} \mathrm{~N}_{0.0165}$ (синий). (Цветной вариант рисунка представлен в электронной версии статьи).

При увеличении концентрации азота в слое $\mathrm{GaAs}_{1-x} \mathrm{~N}_{x}$ постоянная решетки уменьшается, и, соответственно, угловая разница между рефлексом от подложки $\mathrm{GaAs}$ и слоя $\mathrm{GaAs}_{1-x} \mathrm{~N}_{x}$ увеличивается.

После эпитаксиального роста образцы подвергались быстрому термическому отжигу при температурах 760, 780 и $800^{\circ} \mathrm{C}$, длительность составляла 1 мин. Спектры фотолюминесценции (ФЛ) полученных образцов регистрировались при комнатной температуре $(300 \mathrm{~K})$. Установка ФЛ оснащена Nd:YAG-лазером с длиной волны $\lambda=532 \mathrm{HM}$.

\section{3. Результаты и обсуждение}

Были получены спектры фотолюминесценции при комнатной температуре образцов до и после отжига (рис. 2). До отжига на спектрах наблюдается два пика - от подложки GaAs $(1.42$ эВ) и от слоя $\mathrm{GaAs}_{1-x} \mathrm{~N}_{x}$. По мере увеличения температуры отжига меняется форма спектра и увеличивается интенсивность фотолюминесценции. Расщепление спектра фотолюминесценции на серию переходов может объясняться наличием азотных кластеров различной конфигурации в $\mathrm{GaAs}_{1-x} \mathrm{~N}_{x}$, которые возникают в процессе эпитаксиального роста $\mathrm{GaAs}_{1-x} \mathrm{~N}_{x}$ из-за различного размера и электроотрицательности атомов азота и мышьяка.

В [4] было теоретически определено положение азотных пар и других сложных кластеров азота в легированном азотом GaAs: N и GaP:N. Ранее нами экспериментально были продемонстрированы некоторые из этих локализованных состояний в $\mathrm{GaP}_{1-x} \mathrm{~N}_{x}$ [9].

Таким образом, на основании имеющихся данных мы можем предложить различные варианты расположения атомов азота в кристаллической решетке GaAs, энергетические положения которых находятся ниже дна зоны проводимости GaAs, что схематически показано на рис. 3. В общем виде возможные азотные пары обозначаются $\mathrm{NN}_{i}$, при $i=1$ атомы азота располагаются в ближайших анионных положениях.

Таким образом, до отжига в $\mathrm{GaAs}_{1-x} \mathrm{~N}_{x}$ преобладают оптические переходы через локализованные состояния, образованные $3 \mathrm{~N}$ триплетами. По мере отжига образцов $3 \mathrm{~N}$ кластеры распадаются и атомы азота перераспределяются, образуя пары $\mathrm{NN}_{1}$ и $\mathrm{NN}_{4}$. Стоит отметить, что энергетические положения азотных кластеров, наблюдаемые на спектрах фотолюминесценции, совпадают с теоретическими расчетами [4].

Кроме того, были получены рентгенодифракционные кривые качания для образцов после отжига (рис. 4). Для $\mathrm{GaAs}_{1-x} \mathrm{~N}_{x} / \mathrm{GaAs}$ эпитаксиальных структур с различной концентрацией азота форма рентгенодифракционных кривых качания была аналогичной.
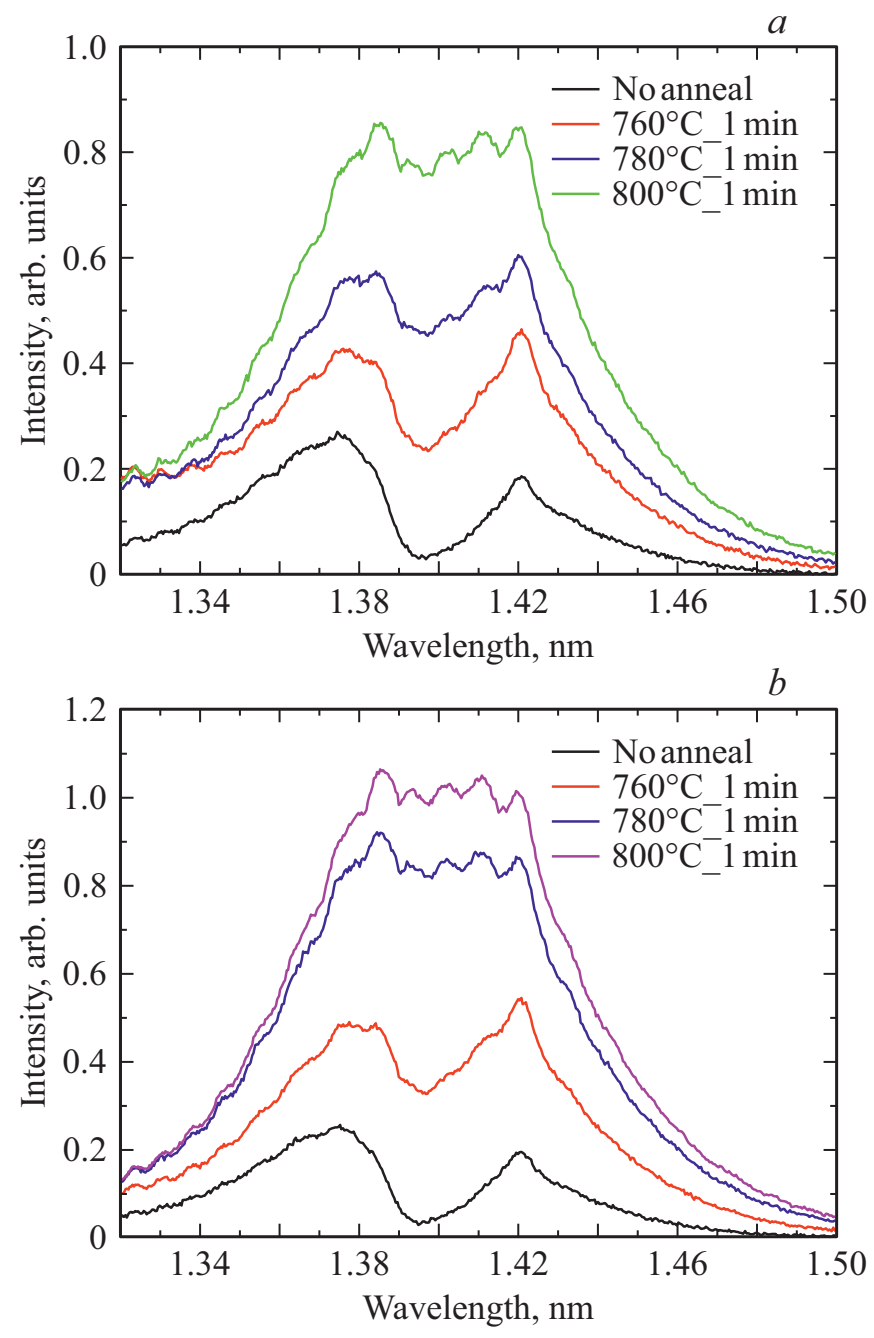

Рис. 2. Спектры фотолюминесценции гетероструктур (a) $\mathrm{GaAs}_{0.9965} \mathrm{~N}_{0.0135} / \mathrm{GaAs}$ и (b) $\mathrm{GaAs}_{0.9935} \mathrm{~N}_{0.0165} / \mathrm{GaAs}$ до отжига и после отжига при температурах 760,780 и $800^{\circ} \mathrm{C}$, полученные при комнатной температуре. 

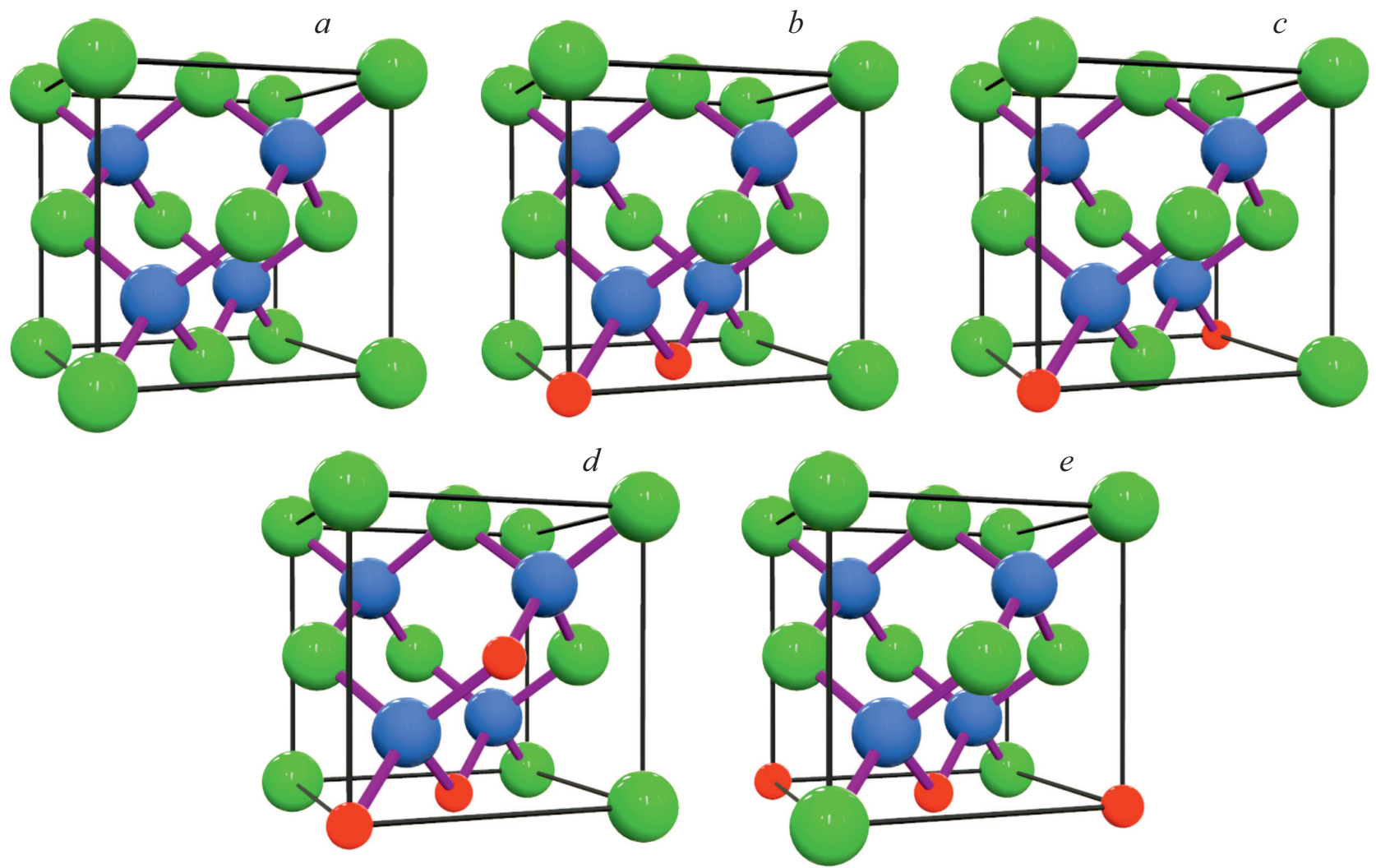

Рис. 3. Расположение атомов азота в решетке GaAs. Зеленым обозначены атомы As, синим - Ga, красным - N. $a-$ GaAs без $\mathrm{N}, b-$ пара $\mathrm{NN}_{1}, c-$ пара $\mathrm{NN}_{4}, d-3 \mathrm{~N}$ триплет, $e-$ цепь атомов азота. (Цветной вариант рисунка представлен в электронной версии статьи).

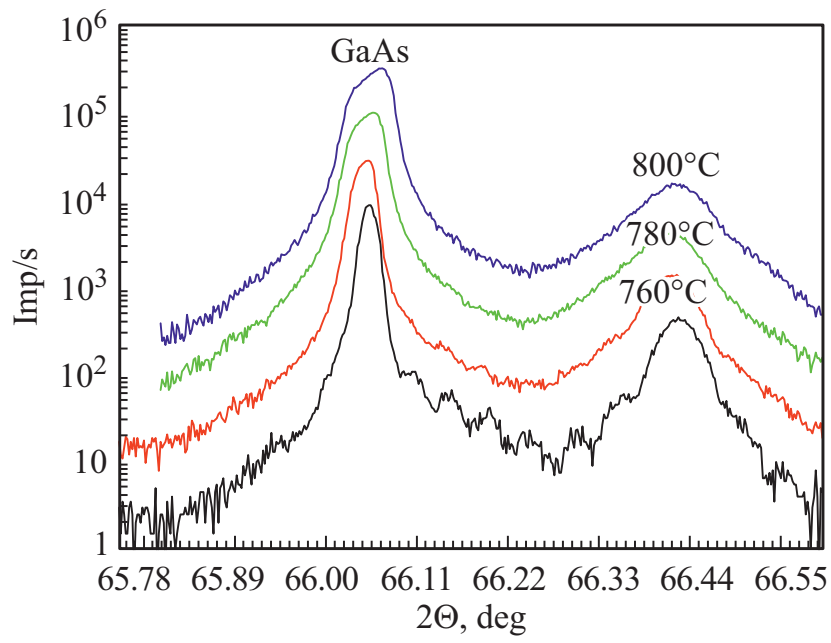

Рис. 4. Рентгенодифракционные кривые качания около симметричного рефлекса GaAs (004). Кривые представлены для образца $\mathrm{GaAs}_{0.9965} \mathrm{~N}_{0.0135} / \mathrm{GaAs}$ до отжига и после отжига при температурах 760,780 и $800^{\circ} \mathrm{C}$.

Видно, что по мере увеличения температуры отжига положение рефлекса от слоя $\mathrm{GaAs}_{1-x} \mathrm{~N}_{x}$ не меняется. Это связано с тем, что при отжиге не происходит де- сорбции азота из-за короткого времени отжига (1 мин), а также достаточно сильной связи $\mathrm{Ga}-\mathrm{N}[10]$.

\section{4. Заключение}

Было исследовано влияние быстрого термического отжига тройных твердых растворов GaAsN/GaAs на распределение атомов азота в кристаллической решетке.

Из-за несоответствия размеров и электроотрицательности атомов азота и мышьяка азот встраивается неравномерно в кристаллическую решетку GaAs. Продемонстрированы варианты расположения атомов азота в кристаллической решетке GaAs.

Показано, что после быстрого термического отжига триплеты $3 \mathrm{~N}$ распадаются и образуются пары $\mathrm{NN}_{1}$ и $\mathrm{NN}_{4}$. Также стоит отметить, что быстрый термический отжиг в диапазоне температур $780-800^{\circ} \mathrm{C}$ длительностью 1 мин не приводит к десорбции азота.

\section{Финансирование работы}

Работа поддержана Министерством образования и науки Российской Федерации (FSRM-2020-0008). 


\section{Конфликт интересов}

Авторы заявляют, что у них нет конфликта интересов.

\section{Список литературы}

[1] J.M. Luthera, S.W. Johnston, S.R. Kurtz, R.K. Ahrenkiel. Appl. Phys. Lett., 88, 263502 (2006).

[2] I. Vurgaftman, J.R. Meyer, N. Tansu, L.J. Mawst. Appl. Phys. Lett., 83, 2742 (2003).

[3] V.K. Kalevich, M.M. Afanasiev, A.Yu. Shiryaev, A.Yu. Egorov. Phys. Rev. B, 85, 035205 (2012).

[4] P.R.C. Kent, Alex Zunger. Phys. Rev. B, 64, 115208 (2001).

[5] Markus Weyers, Michio Sato, Hiroaki Ando. Jpn. J. Appl. Phys., 31, L853 (1992).

[6] S. Francoeur, G. Sivaraman, Y. Qiu, S. Nikishin, H. Temkin. Appl. Phys. Lett., 72, 1857 (1998).

[7] H.A. McKay, R.M. Feenstra, T. Schmidtling, U.W. Pohl. Appl. Phys. Lett., 78, 82 (2001).

[8] Z. Pan, L.H. Li, W. Zhang, Y.W. Lin, R.H. Wu. Appl. Phys. Lett., 77, 1280 (2000).

[9] A.A. Lazarenko, E.V. Nikitina, M.S. Sobolev, E.V. Pirogov, D.V. Denisov, A.Yu. Egorov. Semiconductors, 49, 479 (2015).

[10] S.C, S.-Y. Huang, T.R. Yang. Phys. Rev. B, 64, 113312 (2001).

Редактор Г.А. Оганесян

\section{Influence of rapid thermal annealing on the distribution of nitrogen atoms in GaAsN/GaAs}

A.A. Lazarenko ${ }^{1}$, K.Yu. Shubina ${ }^{1}$, E.V. Nikitina ${ }^{1,2}$, E.V. Pirogov' ${ }^{1}$, A.M. Mizerov ${ }^{1}$, M.S. Sobolev ${ }^{1}$

${ }^{1}$ Alferov University, 194021 St. Petersburg, Russia

${ }^{2}$ loffe Institute,

194021 St. Petersburg, Russia

Abstract The article investigates the effect of rapid thermal annealing of ternary $\mathrm{GaAs}_{1-x} \mathrm{~N}_{x} / \mathrm{GaAs}$ solid solutions on the distribution of nitrogen atoms in the crystal lattice. The samples are studied by photoluminescence spectroscopy and high-resolution $X$-ray diffractometry. Due to the size and electronegativity mismatch of nitrogen and arsenic atoms, nitrogen is incorporated unevenly into the GaAs crystal lattice. Options of the nitrogen atoms arrangement in the GaAs crystal lattice before and after rapid thermal annealing are shown. 